\title{
Gestão de Processos de Negócio em Curso de Sistemas de Informação: Relato de Experiência Utilizando Software Livre
}

\author{
Jessica Lasch de Moura ${ }^{1}$, Gabriel Machado Lunardi ${ }^{1}$, \\ Andrea Schwertner Charão ${ }^{1}$, Patrícia Pitthan Barcelos ${ }^{1}$, Benhur de Oliveira Stein ${ }^{1}$ \\ ${ }^{1}$ Curso de Sistemas de Informação \\ Universidade Federal de Santa Maria - UFSM \\ \{jmoura, glunardi, andrea, pitthan, benhur\}@inf.ufsm.br
}

\begin{abstract}
Resumo. Neste artigo, apresenta-se um relato de experiência de modelagem e implementação de um processo de negócio utilizando o software livre Bonita Open Solution. O processo em questão refere-se a uma atividade-meio em cursos de graduação: a apreciação e validação de atividades complementares realizadas pelos alunos. Sua implantação em um Curso de Sistemas de Informação trouxe melhorias em relação ao processo original e permitiu identificar qualidades e limitações no software livre utilizado. Os artefatos gerados no desenvolvimento deste trabalho encontram-se disponíveis em um repositório público, podendo servir como recurso pedagógico para ensino de Gestão de Processos de Negócio e, também, como solução reutilizável em outros casos com requisitos similares.
\end{abstract}

\begin{abstract}
This article presents an experience report of modeling and implementing a business process using the free software Bonita Open Solution (BOS). The process refers to a support activity in undergraduate courses: the assessment and validation of complementary, extra-curricular activities developed by students. Its implementation in a Bachelor Course in Information Systems brought improvements over the original process and demonstrated some strenghts and limitations of BOS. All artifacts generated in the development of this work are available in a public repository, serving as a pedagogical resource for teaching Business Process Management, as well as a technological solution that can be used in other cases with similar requirements.
\end{abstract}

\section{Introdução}

A gestão de processos de negócio, do inglês Business Process Management (BPM), é um conceito que une as áreas de administração e tecnologia da informação com o objetivo de melhorar processos nas organizações [Weske 2012]. Um processo pode ser entendido como um conjunto de atividades realizadas de forma organizada para atingir um objetivo [ABPMP 2012]. Tais conceitos se alinham com o perfil esperado dos egressos de cursos de bacharelado em Sistemas de Informação, que inclui uma formação "visando o desenvolvimento e a gestão de soluções baseadas em tecnologia da informação para os processos de negócio das organizações de forma que elas atinjam efetivamente seus objetivos estratégicos de negócio” [Conselho Nacional de Educação 2012].

Instituições de ensino superior e, mais especificamente, suas subunidades que gerenciam cursos de graduação, são potenciais "clientes" de BPM, pois realizam processos envolvendo diferentes atores (discentes, docentes, secretários, coordenadores). Tais 
instituições podem se beneficiar com a adoção de ferramentas de apoio à aplicação de BPM, os chamados sistemas de BPM (Business Process Management Systems ou Suites BPMS). Casos e exemplos de gestão de processos de negócio são facilmente encontrados na literatura [Jeston e Nelis 2012], mas geralmente se enquadram no setor empresarial ou de serviços públicos. Em instituições de ensino, a aplicação de BPM ainda é pouco difundida [Barn e Oussena 2010]. Qualquer que seja a área, os artefatos gerados em estudos de caso dificilmente ficam abertos para estudo aprofundado ou reuso em casos similares.

Nesse contexto, surgiu a motivação para a aplicação de BPM a um processo comumente realizado em cursos de graduação e, particularmente, em cursos de bacharelado em Sistemas de Informação. O processo escolhido foi a apreciação e validação de Atividades Complementares, que são realizadas pelos alunos com o objetivo de enriquecer e flexibilizar suas formações. Como tais atividades podem ser realizadas externamente à instituição de ensino, o processo geralmente envolve um trâmite de documentos impressos, entre alunos e coordenação, para que as atividades sejam reconhecidas e computadas na integralização curricular. Possíveis melhorias de tal processo seriam, portanto, a redução de impressões de documentos e maior agilidade na tramitação.

Uma vez analisado o processo, escolheu-se um BPMS que atendesse aos requisitos identificados. Neste trabalho, optou-se pelo Bonita Open Solution (BOS), um software de código aberto que apoia atividades de BPM visando, principalmente, os responsáveis por projetos de desenvolvimento e implantação de aplicações orientadas a processo [BonitaSoft 2012]. Utilizando esta ferramenta, desenvolveu-se um sistema visando à transformação e melhoria do processo, possibilitando que toda a tramitação e apreciação de documentos seja realizada via Web pelos envolvidos.

O presente artigo relata e discute as principais etapas da experiência. Todos os artefatos produzidos durante o desenvolvimento estão disponíveis em um repositório público e podem, assim, ser reusados ou servir como recurso didático. O sistema desenvolvido passou por vários testes com usuários finais e encontra-se, atualmente, implantado no curso de Sistemas de Informação da Universidade Federal de Santa Maria.

O artigo está estruturado da seguinte forma: a seção 2 apresenta uma fundamentação relacionando conceitos de BPM, ferramentas disponíveis a esse propósito e um detalhamento da ferramenta adotada. A seção 3 aborda a legislação educacional sobre atividades complementares em cursos de graduação. Além disso, são explorados os requisitos do processo, com base no caso real analisado. A seguir, a seção 4 relata principais fases do desenvolvimento: modelagem e implementação. A seção 5 faz uma avaliação da experiência e a seção 6 discute trabalhos relacionados. Por fim, a seção 7 traz as considerações finais e perspectivas de trabalhos futuros.

\section{Gestão de Processos de Negócio}

\subsection{Conceitos}

Processos de negócio são atividades cujo objetivo é estabelecer como o trabalho será realizado em uma organização. Pode-se dizer que são ações relacionadas entre si, a fim de promover uma saída [ABPMP 2012]. A gestão (ou gerenciamento) de processos de negócio, comumente designada pela sigla BPM, pode ser definida como um conjunto de boas práticas, com o intuito de mapear e gerenciar processos de negócio, onde se 
envolvem pessoas e atividades automáticas. O principal objetivo é obter uma melhoria desse processo [Weske 2012].

O ciclo de vida de execução de BPM possui várias fases e dentre elas estão a análise, a modelagem e a implementação de processo de negócio. Durante a fase da análise são reunidas informações pertinentes ao processos organizacionais, com a intenção de entendê-los. Também são descritos os objetivos da modelagem e o ambiente de negócio que será modelado. A fase de modelagem é descrita como "um conjunto de atividades envolvidas na criação de representações de um processo de negócio" [ABPMP 2012]. Por fim, a etapa de implementação é definida como a fase que tem o objetivo de realizar o desenho aprovado do processo de negócio, na forma de procedimentos e fluxos de trabalho [ABPMP 2012].

No que diz respeito à modelagem, existe um padrão chamado Business Process Model and Notation (BPMN), que define uma série de elementos padrões para facilitar o entendimento e o desenho do processo [BPMI 2006]. Essa notação engloba termos para atividades, para encarregados de cada tarefa, entre outros. Essa notação é comumente usada por ferramentas BPMS, apresentadas na próxima seção.

\subsection{Ferramentas}

BPMS (Business Process Management System) é o software para utilizar as metodologias do BPM para melhorar um processo. Através do BPMS, pode-se fazer a modelagem do processo e se ter métricas e controles de todo fluxo do processo. Com um software desse tipo, também é possível simular o processo e fornecer relatórios para ajudar durante a tomada de decisões. Ou seja, é possível utilizar essas ferramentas de várias formas visando a melhoria do processo. Alguns exemplos de ferramentas BPMS são Intalio, TIBCO BPM, além soluções Oracle e IBM WebSphere para BPM. Todos esses exemplos são do tipo "software proprietário", que é licenciado com direitos exclusivos para o produtor. Existem, também, ferramentas distribuídas sob licenças de software livre, tais como Activiti, jBPM, ProcessMaker e Bonita Open Solution.

No presente trabalho, optou-se por uma solução de software livre por sua natural flexibilidade e, também, por ser uma solução financeiramente viável para o caso em questão. Após análise da documentação das ferramentas citadas, experimentou-se ProcessMaker e Bonita Open Solution. Esta última foi escolhida pela sua maior habilidade em atender aos requisitos do processo, que serão abordados na seção 3.2.

\subsection{Bonita Open Solution}

Bonita Open Solution (BOS) é uma ferramenta distribuída sob uma licença de software livre, onde o código-fonte é liberado para permitir o uso, cópia, estudo, etc. Trata-se de uma ferramenta desenvolvida em Java, pela empresa BonitaSoft [BonitaSoft 2012].

A ferramenta BOS oferece componentes tanto para a modelagem como para a implementação e transformação de processos. A modelagem e customização de processos é realizada através do Bonita Studio, um componente com interface gráfica tipo desktop que agrupa ferramentas de desenvolvimento. Também é possível agregar funcionalidades aos processos através de conectores, como por exemplo: armazenamento de dados em diversos SGBDs (MySQL, H2, PostgreSQL, entre outros), conexão com servidores de e-mail, repositório de documentos (Alfresco), redes sociais (Facebook, Twitter), geração 
de relatórios (JasperReports), dentre vários outros. Esses conectores, inclusive, podem receber personalizações feitas através de códigos escritos em linguagem Groovy, algo que garante flexibilidade no desenvolvimento.

Junto ao Bonita Studio, tem-se um ambiente de execução de processos chamado Bonita User Experience, permitindo o teste imediato de funcionalidades, num ambiente idêntico ao de um sistema em operação. Nesse ambiente, toda a interação com usuários ocorre via uma interface Web. BOS também disponibiliza um ambiente de execução final, ou seja, para a implantação dos processos em servidores Web baseados em Java como Apache Tomcat e JBoss.

\section{Análise do Processo}

Conforme já mencionado, o foco deste trabalho é o processo que envolve a apreciação de atividades complementares em cursos de graduação, doravante designadas pela sigla ACG (Atividade Complementar de Graduação). O processo é uma atividade-meio (administrativa) que corrobora uma atividade-fim (formação da parte flexível do currículo do aluno). Na sequência, apresenta-se mais informações sobre a legislação educacional que rege tais atividades, para depois partir-se para a análise de um caso real em que o processo em questão é realizado com frequência.

\subsection{Atividades Complementares em Cursos de Graduação}

De acordo com as Diretrizes Curriculares Nacionais para os Cursos de Graduação em Computação [Conselho Nacional de Educação 2012], as atividades complementares são componentes curriculares que têm como objetivo principal enriquecer e expandir o perfil do egresso com atividades que privilegiem aspectos diversos da sua formação, incluindo atividades desenvolvidas fora do ambiente acadêmico.

Desde o ano 2000, tais atividades vêm sendo mencionadas em diretrizes do Conselho Nacional de Educação para diferentes cursos de graduação. No caso de cursos de Sistemas de Informação, as diretrizes estabelecem que tais atividades podem ser cumpridas em diversos ambientes, como a instituição a que o estudante está vinculado, outras instituições e variados ambientes sociais, técnico-científicos ou profissionais, em modalidades tais como: cursos de formação profissional, experiências de trabalho ou estágios não obrigatórios, extensão universitária junto à comunidade, iniciação científica, participação em eventos técnico-científicos, publicações científicas, programas de monitoria e tutoria, representação discente em comissões e comitês, participação em Empresas Júnior, incubadoras, além de diversas outras atividades.

\subsection{Processo Original e Oportunidades de Melhoria}

A presente análise refere-se a um processo real, realizado com frequência no Curso de Sistemas de Informação da Universidade Federal de Santa Maria. Tal curso prevê, para integralização curricular, que o aluno desenvolva um total de 300 horas em Atividades Complementares de Graduação. Essas atividades podem ser desenvolvidas durante os 4 anos de duração do curso e, para que sejam computadas no histórico do aluno, precisam ser comprovadas pelo aluno e apreciadas e validadas junto à coordenação do curso.

Nesse processo, para solicitar o aproveitamento de uma ACG, é necessário que o aluno preencha um formulário e o entregue na secretaria do curso, junto com a 
documentação da atividade (certificados e atestados, por exemplo) e, quando for o caso, um relatório e um parecer do professor responsável. Após esta etapa, segundo normas do curso, a documentação é enviada a um relator, membro do colegiado do curso, para que seja emitido um parecer. Esse parecer é apresentado em uma reunião de colegiado, em que os demais membros também opinam e deliberam sobre a solicitação. Caso a solicitação seja aprovada, a secretaria faz o trâmite necessário para que a carga horária seja computada no histórico do aluno. Algumas solicitações podem seguir um trâmite mais curto, quando se trata de atividades em que o desempenho do aluno não precisa ser avaliado (participações em eventos, por exemplo).

De modo geral, sabe-se que esse processo é trabalhoso, mas entende-se que a apreciação por várias pessoas seja importante para monitorar a qualidade das atividades desenvolvidas pelos alunos. No entanto, o processo manual tem problemas, dentre eles o mau uso do tempo com o trâmite, já que toda a documentação precisa ser entregue em mãos aos envolvidos, um de cada vez, ocasionando atrasos desnecessários. Outro problema é o grande desperdício de papel gerado pela impressão de formulários e documentos.

Para melhoria do processo, o requisito principal é que a interação entre os envolvidos ocorra de forma on-line, via Web, e que os documentos necessários sejam fornecidos e mantidos em formato digital. Deseja-se que todos os envolvidos possam autenticar-se, mesmo fora da instituição, e desempenhar seus papéis no processo, inclusive simultaneamente, amenizando o problema de atraso do processo. Essa autenticação deve aproveitar um cadastro de usuários já existente em uma base LDAP (Lightweight Directory Access Protocol) na instituição. O fluxo de trabalho deve contemplar as etapas de solicitação e apreciação de ACGs. Deseja-se, sempre que possível, que toda a apreciação seja feita através de pareceres on-line, mas em casos onde houverem dúvidas, que o sistema permita encaminhar o processo a uma reunião presencial do colegiado, para facilitar a interação entre os participantes.

\section{Desenvolvimento}

\subsection{Modelagem do Processo}

A etapa de modelagem consistiu na especificação de um fluxo de trabalho seguindo o padrão BPMN, utilizando o Bonita Studio. A figura 1 traz a visão geral do processo modelado. Nela, têm-se as divisões (lanes) "Aluno", "Tutor", "Relator", "Colegiado" e "Coordenação" que delimitam compartimentos de responsabilidade. Em seguida, as tarefas (tasks) representam as atividades realizadas por um ou mais participante, por exemplo "Tutor avalia ACG" ou "Relator avalia ACG". A maioria das tarefas são do tipo human tasks, ou seja, indicam que alguém será responsável por realizar essa tarefa. Uma exceção é a tarefa "Atualiza DB", que é do tipo abstrata (não necessita alguém para iniciá-la), a qual realiza inserção no banco de dados.

Como também mostra a figura 1, cada task é associada a uma etapa do processo, e cada etapa é constituída por formulários Web, os quais devem ser preenchidos pelo usuário correspondente. Por exemplo: o aluno deve descrever a ACG e preencher suas informações de identificação; o professor responsável deve avaliar a solicitação e sugerir alterações, se necessário; o relator do colegiado deve fornecer um parecer sobre tal solicitação e, por fim, o colegiado deve aprovar ou não a solicitação de registro. 


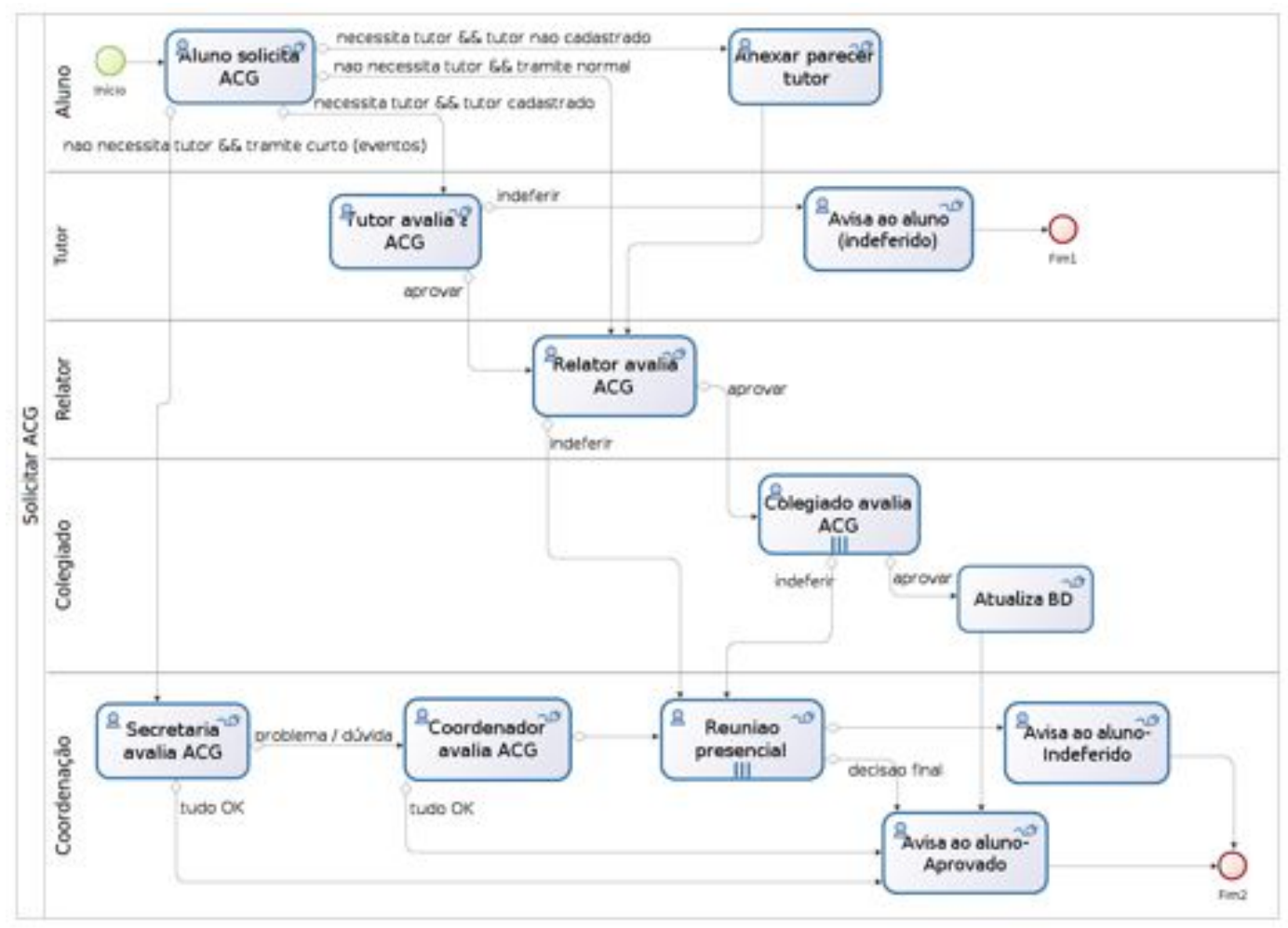

Figura 1. Diagrama da modelagem do processo

$\mathrm{Na}$ etapa de modelagem, a ferramenta BOS satisfez plenamente os requisitos e, no caso em questão, não encontrou-se problemas de expressividade com o padrão BPMN, como relatado em estudos precedentes [Recker et al. 2006, zur Muehlen e Recker 2008].

\subsection{Implementação}

O primeiro passo da implementação foi a criação dos formulários a serem visualizados pelos participantes, conforme os papéis que desempenham. No Bonita Studio, a construção de formulários é facilitada, pois existem vários tipos de elementos pré-definidos que como caixa de texto, campo de data ou uma lista, por exemplo. Utilizando dos elementos citados, é possível construir rapidamente um formulário. Porém, no caso prático em questão, foi necessário realizar algumas customizações através de código na linguagem Groovy, para atender alguns requisitos tais como: geração de uma lista com possíveis professores/tutores, auto-preenchimento das informações dos alunos, entre outros. A figura 2 mostra um exemplo de formulário visualizado pelo aluno, no momento em que este realiza a solicitação de aproveitamento de ACG.

Para que o aluno pudesse anexar os documentos comprobatórios (certificados, atestados, pareceres, etc), utilizou-se um sistema para gestão de conteúdo empresarial, do inglês Enterprise Content Management (ECM), denominado Alfresco. Essa tecnologia foi escolhida por ser suportada via um conector na plataforma BOS, permitindo assim um gerenciamento facilitado dos documentos mesmo após o término dos processos (o que de fato é necessário, pois tais documentos precisam ser arquivados na instituição). Tal como BOS, Alfresco também é um software livre, desenvolvido em Java. 


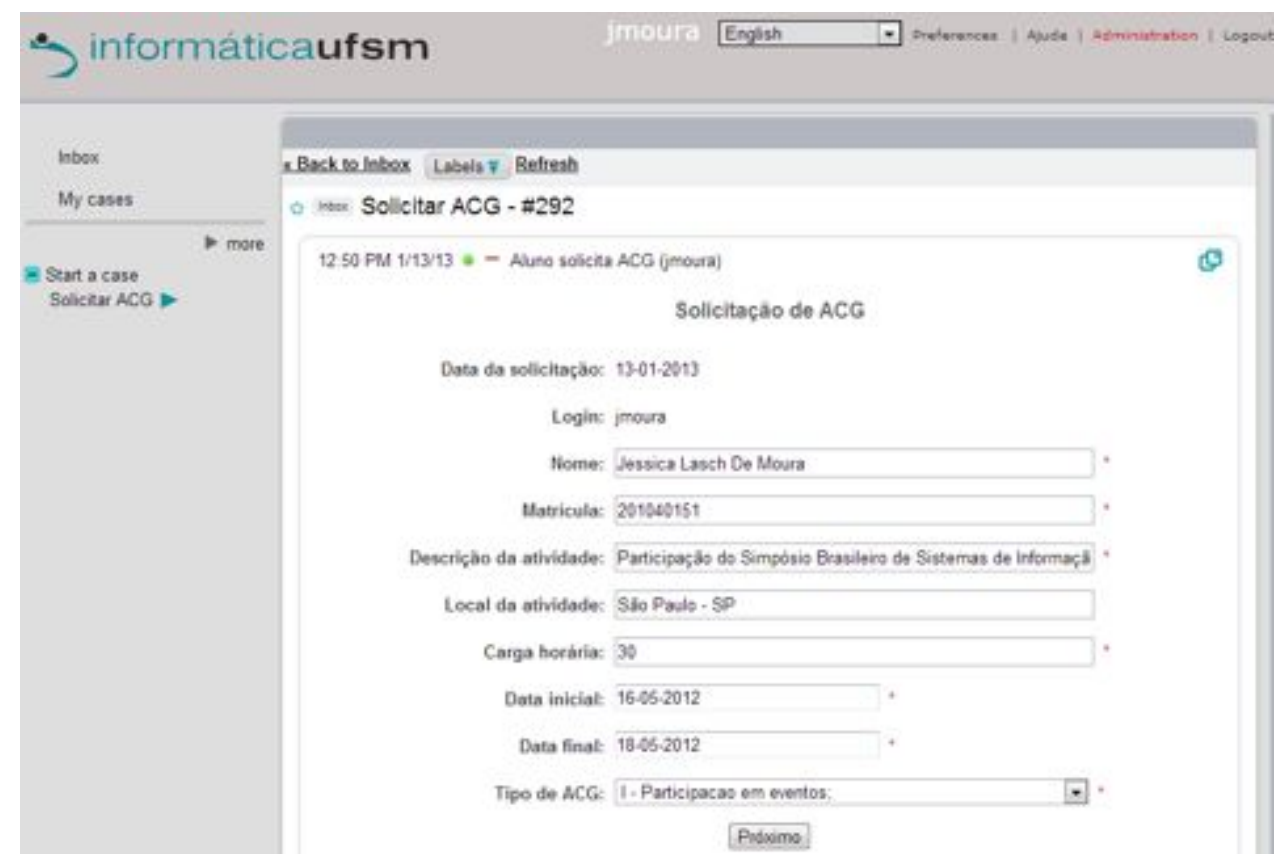

Figura 2. Formulário de solicitação de aproveitamento

Para implementar a autenticação via LDAP, foi criado um novo módulo de login para o BOS, baseado em códigos Java retirados dos fóruns de discussão da ferramenta. Esse módulo substitui o módulo padrão de login, o qual autentica usuários através de um banco de dados interno à plataforma de execução. Paralelamente, foi necessário cadastrar grupos de usuários diretamente no BOS, pois muitas atividades dependiam dessa vinculação de usuários a grupos, e tais grupos não existiam na base LDAP. Os grupos criados foram "tutores", "relatores", "colegiado" e "coordenação", correspondendo às divisões de responsabilidade no processo. Também foi necessário vincular usuários ao grupo "admin", que é um grupo padrão no BOS para designar usuários com permissões administrativas sobre os processos.

Outro ponto importante na implementação do processo foi a geração de relatórios voltados tanto para alunos como para a coordenação, tais como histórico de solicitações de ACGs e resumo de carga horária homologada e faltante ao currículo do aluno. A ferramenta BOS possui um tipo de conector que utiliza a biblioteca JasperReports para a geração de relatórios associados a um processo. Tal conector busca os dados do relatório em uma base de dados externa à base do BOS.

A partir da necessidade de relatórios, portanto, foi preciso definir uma base de dados externa para o armazenamento das informações de solicitação/apreciação de atividades. Iniciou-se definindo a estrutura relacional contendo 5 tabelas ${ }^{1}$. Em seguida, foi escolhido o SGBD Java H2 pela simplicidade e eficiência. Depois disso, foi necessário incluir conectores do SGBD $\mathrm{H} 2 \mathrm{em}$ tasks do processo, com as respectivas consultas SQL, para que a base de dados de atividades ficasse constantemente atualizada.

Para a implementação dos relatórios, foi necessário construir uma definição XML

\footnotetext{
${ }^{1}$ Estrutura disponível no repositório: http://code.google.com/p/bpm-infufsm/
} 
(contendo as consultas SQL, definições de campos e formatações) para cada relatório utilizando a ferramenta gráfica iReport. Mais tarde, essas definições são executadas pela biblioteca JasperReports também provida por Bonita através do conector, contra uma fonte de dados. Para que os usuários tivessem acesso aos relatórios gerados, foram criados mais dois processos chamados "Listar ACGs do usuário" (histórico) e "Resumo ACGs do usuário" (carga-horária). A figura 3 ilustra esse mecanismo de funcionamento básico de conectores BOS para geração de relatórios com a biblioteca JasperReports.

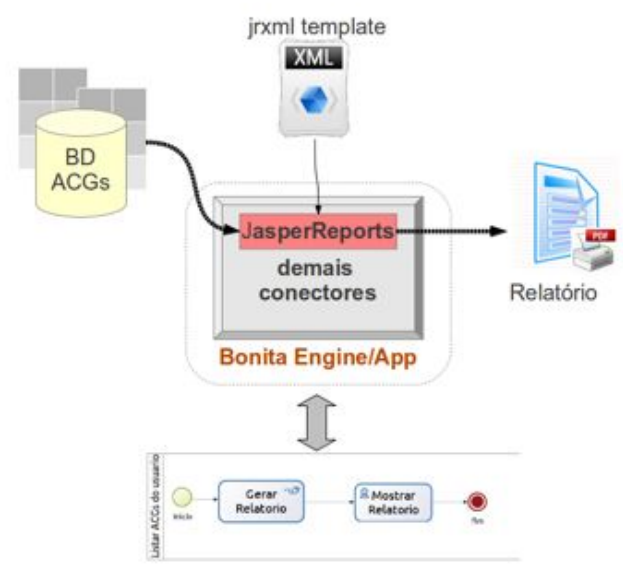

Figura 3. Funcionamento do conector que gera relatórios

A última etapa da implementação foi a personalização da interface Bonita User Experience (versão 5.7.2), substituindo imagens e cores padrão do ambiente por elementos que remetem à identidade visual da instituição. Esta customização foi realizada alterando-se arquivos HTML e CSS que definem a interface Web do BOS. Outras personalizações mais profundas também foram necessárias na interface, para remover componentes julgados desnecessários, que acabavam por confundir os usuários. Porém, estas alterações não poderiam ser feitas apenas editando-se imagens e arquivos. Neste caso, foi necessário a alteração do código do próprio BOS, algo que só foi possível pelo fato de a ferramenta ser distribuída como software livre.

Ao longo do desenvolvimento, foi criado um repositório on-line, disponível em http://code.google.com/p/bpm-infufsm/. Nesse repositório, encontram-se todos os artefatos produzidos durante o desenvolvimento do trabalho (requisitos, modelo do processo, versões do código implementado, esquemas de banco de dados). O repositório também permite que usuários registrem dúvidas, problemas ou sugestões.

\section{Implantação e Avaliação}

Após a implementação foi iniciada a fase de testes com usuários finais (membros do colegiado e alguns alunos bolsistas). Os testes realizados ao longo do desenvolvimento indicaram aceitação por parte dos usuários e revelaram oportunidades de melhoria da interface e da modelagem do processo. Após várias revisões, teve início a fase de implantação do processo, permitindo avaliá-lo em relação aos objetivos iniciais. 
O processo foi disponibilizado à comunidade do curso, composta por cerca de 120 alunos e 25 professores. O colegiado do curso, seguindo normas internas, fixou uma data limite para registro de atividades já realizadas pelos alunos. Logo no primeiro mês, o sistema registrou mais de 100 instâncias (casos abertos) do processo. Em consultas informais aos usuários, logo no início da implantação, notou-se boa aceitação da abordagem utilizada, que evita trâmite de documentos em papel. A experiência com BOS no caso em questão foi apresentada a alunos de uma disciplina sobre modelagem de processos de negócio, suscitando interesse e reconhecimento.

No segundo mês de uso do sistema, buscou-se quantificar a aceitação do sistema junto aos alunos. A decisão de iniciar a avaliação pela experiência dos alunos justifica-se pois estes, além de formarem a maioria dos usuários, são os atores que iniciam o processo solicitando uma ACG. Para esta avaliação, elaborou-se um questionário com questões de múltipla escolha, cada uma com 5 opções de resposta baseadas na escala de Likert ${ }^{2}$. Além de questões sobre aceitação, o questionário tinha também outras questões relacionadas a prioridades na implementação de correções e melhorias, incluindo uma questão discursiva opcional. Ao todo, 29 alunos responderam ao questionário, que era anônimo e foi enviado aos alunos por e-mail. A tabela 1 resume os resultados obtidos para as questões sobre aceitação do sistema. As respostas foram agrupadas em 3 categorias, buscando contrastar opiniões em concordância ou discordância.

Tabela 1. Resultados do questionário de aceitação

\begin{tabular}{l|l|l|l}
\hline Questão & $\begin{array}{l}\text { Concordo } \\
\text { totalmente ou } \\
\text { parcialmente }\end{array}$ & Indiferente & $\begin{array}{l}\text { Discordo } \\
\text { totalmente ou } \\
\text { parcialmente }\end{array}$ \\
\hline $\begin{array}{l}\text { O trâmite on-line é melhor do que } \\
\text { o trâmite em papel. }\end{array}$ & 15 & 2 & 12 \\
\hline $\begin{array}{l}\text { O sistema das ACGs é um bom exemplo de } \\
\text { TI aplicada a um problema real. }\end{array}$ & 22 & 2 & 5 \\
\hline $\begin{array}{l}\text { A integração do sistema com a base de } \\
\text { usuários existente é conveniente. }\end{array}$ & 24 & 3 & 2 \\
\hline $\begin{array}{l}\text { O sistema on-line das ACGs é fácil } \\
\text { de aprender a usar. }\end{array}$ & 11 & 4 & 14 \\
\hline
\end{tabular}

Observando-se a tabela 1, nota-se que houve concordância em alguns aspectos, mas que a aceitação da abordagem não foi tão forte quanto se esperava. Em particular, houve um número significativo de usuários que não consideraram o sistema fácil de aprender a usar. Além disso, muitos não consideraram o trâmite on-line melhor do que o trâmite em papel.

Para melhor interpretar esses resultados, é necessário saber que o questionário foi aplicado próximo à data limite de registro de ACGs, período em que houve um aumento considerável no número de casos abertos no sistema. Além disso, o sistema apresentou falhas nesse período, algumas delas ainda em investigação. Essas falhas e outras limitações observadas são discutidas na próxima seção.

\footnotetext{
${ }^{2}$ Opções: Concordo totalmente, Concordo parcialmente, Indiferente, Discordo parcialmente, Discordo totalmente
} 


\subsection{Limitações}

Um dos principais problemas observados diz respeito a casos que falham, por exemplo devido a alguma entrada não prevista ou devido a problemas de comunicação com o gerenciador de documentos (Alfresco) ou com o banco de dados. Na implementação atual, os casos falhos são simplesmente assinalados aos usuários, sem uma explicação para isso. No questionário de avaliação, muitos usuários sugeriram prioridade máxima na resolução deste problema. Entretanto, a identificação do motivo da falha só é possível pela análise de $\log s$ do BOS, sendo restrita a administradores e muitas vezes difícil de localizar. É possível alterar a modelagem para incluir caminhos alternativos em caso de falhas, mas isso torna o processo mais complexo e não resolve todos os tipos de problemas (por exemplo, bugs no processo). Tudo isso revela a importância de se realizar muitos testes com o processo. No entanto, testes automatizados não são suportados pela ferramenta, dificultando o trabalho.

Ainda com relação a falhas, observou-se em diversas situações que os serviços do sistema não foram corretamente restabelecidos após serem interrompidos. Por exemplo, houve necessidade de recuperação manual do banco de dados interno do BOS, após uma sequência de quedas de energia. Em outra situação, um arquivo de lock não removido ocasionou degradação significativa no tempo de resposta para visualização de formulários no sistema.

A interface administrativa do BOS, mostrada na figura 4, também revelou algumas limitações. Por exemplo, quando se tem uma grande quantidade de casos abertos, torna-se difícil organizá-los ou localizar um caso específico. Em uma versão comercial do software (Bonita Subscription Pack) existe a opção de filtragem e busca dos casos, mas isso não está disponível no BOS. Porém, com a experiência adquirida, estima-se que o problema possa ser resolvido através de relatórios.

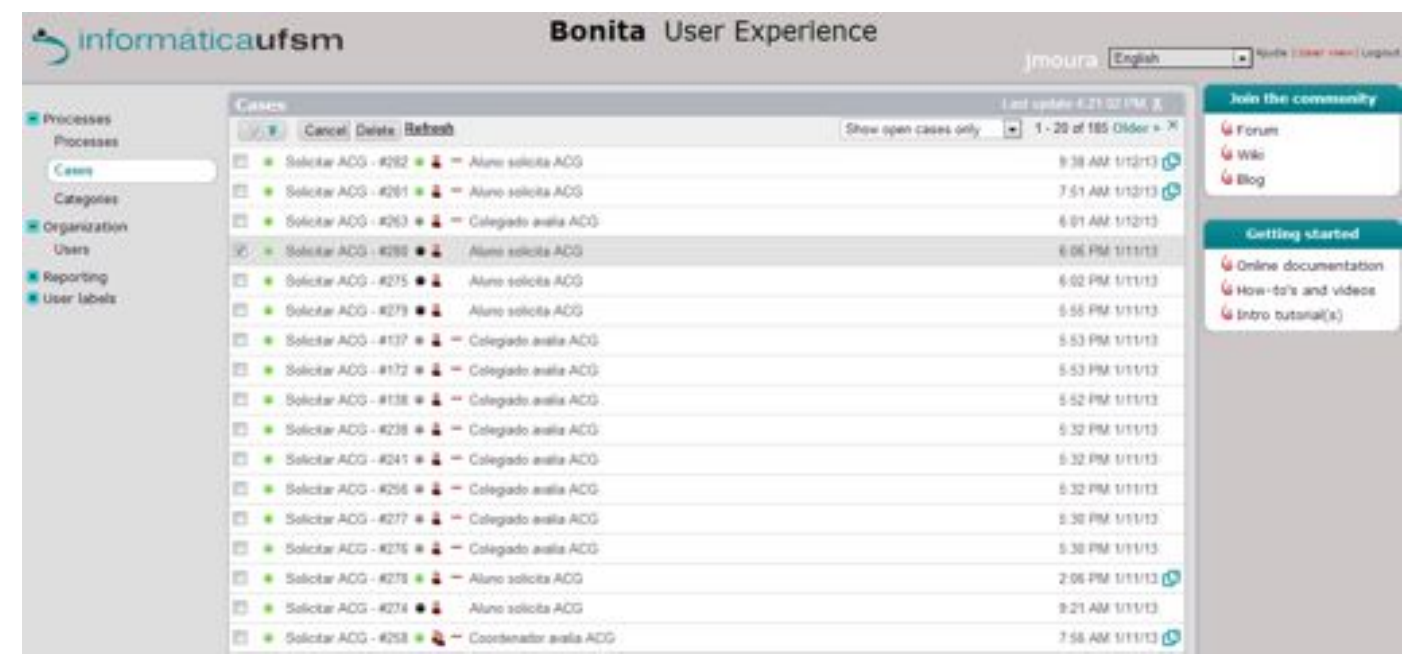

Figura 4. Interface Administrativa

Por fim, é importante ressaltar que alguns recursos do BOS possuem documentação escassa (por exemplo, integração com LDAP e geração de relatórios) e só puderam ser utilizados com auxílio do fórum de discussão da ferramenta, consumindo 
tempo significativo de desenvolvimento. Uma necessidade que surgiu durante o trabalho foi a edição colaborativa de um processo, já que a equipe tem mais de um desenvolvedor. Isso é previsto em uma versão comercial do software mas, novamente, não está incluído no BOS. Mesmo assim, foi possível criar uma estratégia manual de compartilhamento e controle de versão com Subversion, utilizando um cliente e um servidor externos.

\section{Trabalhos Relacionados}

Com o crescente interesse em tecnologias de BPM, é comum encontrar estudos de caso em páginas Web de empresas que comercializam soluções ${ }^{3}$. Na literatura técnicocientífica, muitos autores têm se dedicado a investigar a eficácia das soluções, principalmente no que diz respeito ao uso da notação BPMN para modelagem de processos [Recker et al. 2006, zur Muehlen e Recker 2008, Ostroski et al. 2012]. Estudos envolvendo outras fases e ferramentas de BPM são menos frequentes, embora se reconheça sua importância [Sordi e Spelta 2007, Wohed et al. 2009].

No trabalho de Barn e Oussena (2010), encontra-se um estudo de caso de BPM aplicado a instituições de ensino superior no Reino Unido. O processo estudado, neste caso, tem por objetivo a validação de projetos de cursos superiores, resultando na criação de novos cursos ou na continuidade de cursos existentes. A modelagem do processo é feita com a ferramenta Intalio Designer e os autores relatam desafios enfrentados nesta etapa. Nosso trabalho relaciona-se a este, à medida que explora BPM no contexto de instituições de ensino superior e aborda qualidades e limitações de uma ferramenta. Porém, nosso trabalho abrange mais etapas de BPM, chegando à implantação e avaliação por usuários finais.

Analisando-se eventos e periódicos que publicam relatos de experiências na área, não encontrou-se outros trabalhos associando, ao mesmo tempo, a utilização de software livre para BPM e sua aplicação a uma atividade meio no ambiente acadêmico.

\section{Considerações Finais}

Este artigo abordou uma experiência de aplicação de conceitos de BPM e ferramentas BPMS, inserido na realidade de cursos de graduação. Como contribuições, o trabalho traz a modelagem e implementação de um sistema baseado em tecnologias de BPM, visando melhorar um processo que é realizado com bastante frequência em uma instituição de ensino superior e, mais especificamente, no âmbito de um curso de Sistemas de Informação. O sistema desenvolvido foi implementado levando em conta o processo de apreciação de ACGs em uma instituição específica, mas está disponível em um repositório e pode ser adaptado para outras instituições de ensino superior submetidas à mesma legislação.

Outra contribuição deste trabalho está na exploração da ferramenta Bonita Open Solution. A experiência permitiu identificar qualidades da ferramenta, principalmente na modelagem do processo e nas suas possibilidades de extensão, customização e conexão com outros serviços. Também foi possível identificar algumas limitações que só ficaram claras com a implantação do sistema.

Como trabalhos futuros, pretende-se dar continuidade na exploração de BPM e suas contribuições para melhoria de processos internos ao curso, ampliando a experiência.

\footnotetext{
${ }^{3}$ Ver por exemplo: http://www.orbis-software.com/customers/
} 
De modo mais geral, um aspecto que merece ser explorado é o teste automatizado de processos, que ainda carece de suporte adequado na ferramenta utilizada.

\section{Referências}

ABPMP (2012). Guide to the Business Process Management Body of Knowledge (BPM $C B O K)$. Association of Business Process Management Professionals, 2nd edition.

Barn, B. e Oussena, S. (2010). BPMN, toolsets, and methodology: a case study of business process management in higher education. In Information systems development: towards a service provision society, pages 685-693. Springer, Boston, MA.

BonitaSoft (2012). Bonitasoft open source workflow and bpm software. Disponível em: http://www.bonitasoft.com.

BPMI (2006). OMG: Business Process Modeling Notation specification. Final Adopted Specification. Object Management Group. Disponível em: http://www.bpmi.org/.

Conselho Nacional de Educação (2012). Parecer cne/ces no: 136/2012. conselho nacional de educação/câmara de educação superior. diretrizes curriculares nacionais para os cursos de graduação em computação. processo no $23001.000026 / 2012-95$.

Jeston, J. e Nelis, J. (2012). Business Process Management: Practical Guidelines to Successful Implementations. Butterworth-Heinemann, 2nd edition.

Ostroski, E. H., Digiampietri, L. A., Prado, E. P. V., de Albuquerque, J. P., Frazão, J. B., Errante, P. R., e Condino-Neto, A. (2012). Avaliação de BPMN para caracterização estratégia de uma organização de pesquisas acadêmicas. In VIII Simpósio Brasileiro de Sistemas de Informação, pages 210-221.

Recker, J., Indulska, M., Rosemann, M., e Green, P. (2006). How good is BPMN really? Insights from theory and practice. In Proceedings of the 14th European Conference on Information Systems.

Sordi, J. O. d. e Spelta, A. G. (2007). Análise de componentes da tecnologia de Business Process Management System (BPMS) sob a perspectiva de um caso prático. JISTEM - Journal of Information Systems and Technology Management, 4:71 - 94.

Weske, M. (2012). Business Process Management: Concepts, Languages, Architectures. Springer, 2nd edition.

Wohed, P., Russell, N., Ter Hofstede, A., Andersson, B., e Van Der Aalst, W. (2009). Patterns-based evaluation of open source BPM systems: The cases of jBPM, OpenWFE, and Enhydra Shark. Information and Software Technology, 51(8):11871216.

zur Muehlen, M. e Recker, J. C. (2008). How much language is enough? Theoretical and practical use of the Business Process Modeling Notation. In Proceedings of the 20th International Conference on Advanced Information Systems Engineering. 